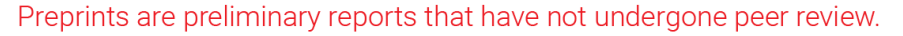 \\ They should not be considered conclusive, used to inform clinical practice, \\ or referenced by the media as validated information. \\ UVB-Induced Nuclear Translocation of PTEN Promotes Radiation Sensitivity of Human Malignant Glioma U87MG Cells
}

\section{Wei Zhao}

Henan University

Chao Yao

Henan University

\section{Yang An}

Henan University

\section{Yuting Jia}

Henan University

\section{Zhenhua Song}

Henan University

Leilei Sun

Henan University

Shaoping Ji

Henan University

\section{Li Ma}

Henan University

Fengling Wang ( $\sim$ wangfl@henu.edu.cn )

Henan University https://orcid.org/0000-0002-4125-9188

\section{Research}

Keywords: PTEN, ultraviolet radiation, nucleoplasm distribution, U87MG cells

Posted Date: October 16th, 2020

DOl: https://doi.org/10.21203/rs.3.rs-88310/v1

License: (c) (i) This work is licensed under a Creative Commons Attribution 4.0 International License.

Read Full License 


\section{Abstract}

\section{Background}

PTEN is a tumor suppressor gene, which is often inactivated through mutation and/or deletion in diverse human tumors. In the cytoplasm, PTEN negatively regulates the phosphatidylinositol 3' kinase (PI3K) signaling pathway, while PTEN also shuttles to the nucleus, where PTEN stabilizes genome and/or participates in DNA repairment after damage. PTEN's subcellular localization seems to be regulated by various factors.

Methods

U87MG cells with expressing Wild-type-PTEN (WT-PTEN) and K13E-PTEN mutant were engineered respectively. The relationship between UV radiation and PTEN's subcellular localization were elucidated using nuclear and cytosolic fractionation and fluorescence co-localization assay. Related signaling pathways were studied with western blot assays.

Results

Here, we demonstrated that UV irradiation could promote nuclear translocation of both WT-PTEN and K13E-PTEN mutant in a dose-dependent manner, but WT PTEN had a higher level of nuclear accumulation after inducing by UV-irradiation compared to K13E-PTEN. In contrast, the cytoplasmicnuclear translocation of exogenous PTEN was critical to its tumor-suppressing functions that made U87MG cells more sensitive to the UV irradiation.

\section{Conclusion}

Our findings may have implications for further revealing the function of nuclear PTEN and provide insights for clinical treatment of PTEN-deficient tumors.

\section{Introduction}

PTEN is a tumor suppressor gene located on the human chromosome 10, which was thought to be expressed primarily in the cytoplasm in many cells [1-3]. It has been reported that PTEN is also distributed in the cell nucleus of many normal and cancerous cells, which may have growth-regulatory functions via signaling mechanisms that are distinctive from those of cytoplasmic PTEN [4]. The levels of PTEN in the cytoplasm and nucleus are directly associated with its tumor suppressor functions, and quantitatively monitoring PTEN expression in the cytoplasm and nucleus may contribute to the prediction of cancer susceptibility [5].

Though PTEN has no typical nuclear protein signal, it can shuttle between nucleus and cytoplasm by relying on certain mechanisms, such as ubiquitination, which can result in PTEN nuclear translocation, leading to regulation in the physiological or pathological processes such as cell proliferation, apoptosis 
and its tumor suppressing ability [6-8]. Cytoplasmic PTEN, was essential to antagonize the PI3K pathway and inhibit the proliferation, invasion and migration of tumor cells [9]. Nuclear PTEN, by contrast, was found to participate in DNA repairing and nuclear stability [7]. Furthermore, it was indispensable for PTEN function to have right $\mathrm{C} 2$ domain, of which (PTEN-4A) interacts with the transcription factor E2F1 to lose its inhibitions in lung cancer [10]. Several mechanisms for the nucleocytoplasmic translocation of PTEN have been proposed, including passive diffusion through nuclear pores [11]; a Ran-dependent mechanism [12]; depending on Major Vault Protein [13]; mono-ubiquitin pathway involved in E3 ligase NEDD4-1 [9,14]; SUMOylation mechanism [15]. K13 and K289 are the two critical conserved sites of PTEN ubiquitin modification, if which are mutated (K13E and K289E), PTEN will be accumulated mainly in the cytoplasm, resulting in impaired tumor suppressive ability of PTEN in human glioblastoma [16]. While the mechanism, function and relevance of nuclear PTEN localization remain to be further elucidated.

Solar Ultraviolet Radiation (UVR) can induce skin Cancers [17], medium-wave ultraviolet (UVB) is a part of sunlight with a wavelength from $280 \mathrm{~nm}$ to $320 \mathrm{~nm}$, which can cause DNA damage, promote apoptosis of retinal pigment epithelium (RPE) cells [18] 『leading to DNA damage and repairment [19]. In addition, UVB irradiation resulted in a decreased PTEN expression in RPE cells, which triggered DNA damage and increased UVB-induced apoptosis via activating p53-dependent pathway [18]. However, the relationship between UVR and nuclear location of PTEN, has been rarely reported to date.

Therefore, we selected human glioma PTEN-null U87MG cells to establish both WT-PTEN and K13E-PTEN expressing cell models. The results of the experiment demonstrated that UV irradiation can promote nuclear entry of both WT-PTEN and K13E-PTEN mutant in a dose-dependent manner. And the enhanced nuclear translocation ability further contributed to radio-toxicity of UVR, leading to increased irradiation sensitivity of U87MG cells. Our findings may have implications for further demonstrating the likely relationship between radiation and PTEN function.

\section{Materials And Methods}

\section{Cell culture and UVB treatment}

HEK293T and U87MG cells were purchased from ATCC, and were cultured in DMEM medium with 10\% Fetal Bovine Serum (FBS) and $1 \%$ Penicillin/Streptomycin at $37{ }^{\circ} \mathrm{C}$ with $5 \%$ of $\mathrm{CO}_{2}$. Lentiviral plasmids (LV5 and LV8) carrying human GFP-WT-PTEN and RFP-K13E-PTEN ORF respectively were preserved in our laboratory. The packing and purification of the recombinant lentiviral vectors were performed by the Gene Pharma Company (Shanghai, China). The indicated U87MG cells were infected with the recombinant lentiviral vectors, and the infection efficiency was observed with a fluorescence microscope. Subsequently, the infected cells were selected with puromycin for 2 weeks.

In addition, the HEK293T cells were transiently transfected with pEGFP-NI-K13E-PTEN, pEGFP-NI-WTPTEN or empty plasmid using a lipofectamine 2000 method, according to manufacturer's protocol. For UVB exposure, the SH-2 UVB phototherapy instrument (Sigma, Shanghai) with radiation intensity of 15 
$\mathrm{mW} / \mathrm{cm}^{2}$, and the irradiation area of $22 \mathrm{~cm}^{2}$ was used. Before UVB treatment, the instrument was sterilized with alcohol and positioned $20 \mathrm{~cm}$ above the dish. The cells were washed with PBS twice, followed by exposing to different doses of UVB $\left(0,90,180,270 \mathrm{~mJ} / \mathrm{cm}^{2}\right)$ in PBS to avoid the photosensitization effect of culture medium components on the cells. After UVB treatment, the cells were continued to culture at $37^{\circ} \mathrm{C}$ for a certain time.

\section{Nuclear and cytosolic fractionation}

Cytosolic and nuclear proteins were extracted from cell homogenates using a Nuclear/Cytosol Fractionation Kit (Biyuntian Biological Co., Ltd, China) according to the manufacturer's instructions. Briefly, cells were centrifuged at $800 \mathrm{~g}$ for $3 \mathrm{~min}$ at $4{ }^{\circ} \mathrm{C}$. The pellet added reagent $A$ with fresh PMSF was vortexed for $10 \mathrm{~s}$, and placed on ice for $15 \mathrm{~min}$. The reagent $\mathrm{B}$ was added, vortexed for $10 \mathrm{~s}$ and placed on ice for $1 \mathrm{~min}$. After samples were centrifuged at $13000 \mathrm{~g}$ for $5 \mathrm{~min}$ at $4^{\circ} \mathrm{C}$, the supernatant fraction was collected as cytosolic fraction. The pellet containing nuclear protein was added nuclear protein extraction reagent with PMSF, and then the mixture was vortexed $30 \mathrm{~s}$ and placed on ice for 30 min, repeated once every 2 min to ensure that the cell pellet was completely dispersed. Then the samples were centrifuged at $13000 \mathrm{rpm}$ for $10 \mathrm{~min}$ at $4^{\circ} \mathrm{C}$. and the supernatant was taken as the nuclear fraction for immediate use or stored at $-80^{\circ} \mathrm{C}$.

\section{MTT Assay}

Different groups of U87MG cells including parental U87MG cells, vector control U87MG cells, PTENexpressing U87MG cells and K13E-PTEN expressing U87MG cells were respectively seeded in 96-well plates at a density of 10000 cells per well and allowed to adhere for $24 \mathrm{~h}$. Then the cells were exposed to different doses of UVB $\left(60,120,240,480\right.$ and $\left.960 \mathrm{~mJ} / \mathrm{cm}^{2}\right)$ respectively, and MTT assay was performed after $24 \mathrm{~h}$ post-UVR culture. Cells were then incubated for $4 \mathrm{~h}$ with $5 \mathrm{mg} / \mathrm{ml}$ of MTT, dissolved in serum free DMEM medium. Discarding the mixture was followed by the addition of $150 \mu \mathrm{L}$ DMSO, incubating for $10 \mathrm{~min}$ in the dark. The absorbance was measured at $490 \mathrm{~nm}$ using microplate reader (SpectraMax M2, USA).

\section{Western blotting}

Western blotting was performed as described previously [20,21]. Antibodies used were as follows: antiPTEN (\#9188, Cell Signaling Technology), anti-Caspase-3 antibody (\#AC030, Beyotime), anti-caspase-3 cleaved at Asp175 (\#AC033, Beyotime), anti-GAPDH (\#AG019-1, Beyotime), anti- $\beta$-tubulin (\#10094-1-AP, Proteintech) and anti-Lamin B (Proteintech).

For immunoblotting, cells were lysed in RIPA buffer containing $1 \times$ cocktail protease inhibitor for 30 min on ice followed by centrifugation at $14,000 \mathrm{~g}$ for $5 \mathrm{~min}$. Lysates were heated to $100^{\circ} \mathrm{C}$ in SDS sample buffer with $50 \mathrm{mM}$ DTT for $10 \mathrm{~min}$, resolved by SDS-PAGE, and electroblotted onto PVDF membrane (Millipore, Billerica, USA). PVDF membrane were then blocked in 5\% milk in TBS with $0.1 \%$ Tween-20 and probed with the monoclonal antibodies. After incubation with the secondary antibody, chemiluminescence 
solution was added onto membrane, and reactive bands visualized using Chemiluminescence imagers (Protein Simple, PE0684, USA).

\section{Fluorescence co-localization Assay}

HEK293T cells were transiently transfected with pEGFP-NI-K13E-PTEN, pEGFP-NI-WT-PTEN or empty plasmid in 6-well plates at a density of $3110^{5}$ cells per well and allowed to adhere for $24 \mathrm{~h}$. Then the cells were exposed to different doses of UVB $\left(0,90,180\right.$, and $\left.270 \mathrm{~mJ} / \mathrm{cm}^{2}\right)$ respectively, and DAPI staining was performed after $6 \mathrm{~h}$ or $12 \mathrm{~h}$ post-UVR culture. Briefly, cells were washed 3 times with PBS buffer, followed by the addition of $500 \mu \mathrm{L} 4 \%$ paraformaldehyde, incubated for $30 \mathrm{~min}$ in the dark. Next, the cells were washed with PBS 3 times and subsequently stained with DAPI $(5 \mathrm{mg} / \mathrm{mL})$ in PBS for $15 \mathrm{~min}$ in the dark. After washing with PBS buffer for 3 times, cells were observed with fluorescent microscope (Japan, Nikon, DS-Qi2) and the images were captured using the NIS-Elements AR software. Green is GFP-PTEN, Blue is DAPI-labeled nucleus, and the overlapped blue and green in the merged images represents PTEN localization in the nuclear. Nuclear GFP-PTEN were quantified in at least 50 cells. The relative nuclear GFP-PTEN level in each group was expressed as a ratio between the numbers of nuclear GFP-PTEN and the total counted cells.

\section{Statistical Analyses}

Statistical analysis was performed for all data by using Student's t-test and a value of $p<0.05$ was considered significant. All tests were performed using GraphPad Prism 7 software.

\section{Results}

\section{Establishment of PTEN expression in PTEN-deficient cell lines}

Lentiviral expression vector (LV5 and LV8) respectively carrying human WT-PTEN and K13E-PTEN ORF were preserved in our laboratory. Then PTEN-deficient glioblastoma cell line U87MG (Fig. 1A) were infected respectively with lentivirus expressing WT-PTEN, mutant K13E-PTEN, or empty vector, where a greater transfection efficiency was observed by fluorescence microscopy (Fig. 1B). Then WT-PTEN/K13EPTEN expression in U87MG cells were established by puromycin selection. And overexpression of WTPTEN and mutant K13E-PTEN in U87MG cells was strongly detectable by Western blot analysis (Fig. 1C), which suggesting a successful establishment for these two cell models.

\section{Subcellular localization of PTEN and mutant}

Subcellular localization of the wild-type and mutant gene products in U87MG cells was examined by western blotting of the cytoplasmic and nuclear fractions (Fig. 2A). Corresponding cytoplasmic and nuclear protein biomarkers, Tubulin and Lamin B were selected to identify the successful separation of the respective cytoplasmic and nuclear fractions. As illustrated in Fig. 2A, the WT-PTEN was expressed both in cell nucleus and cytoplasm, and mainly presented in the nucleus of overexpressing U87MG cells. 
While for the K13E-PTEN expression, PTEN staining was weak in the nucleus and conversely, it was primarily observed in the cytoplasm. Consistent with the result of the cytoplasmic and nuclear fractions, the fluorescence microscope analyses of the transiently transfected HEK293T cells also showed that WTPTEN exhibited a more distribution in nuclear than cytoplasm compartment, while K13E-PTEN primarily presented in the cytoplasm (Fig. 2B). These results supported the idea that as one of the main conserved sites of PTEN ubiquitin, if K13 of PTEN is mutated to glutamic acid (K13E), PTEN accumulates mainly in the cytoplasm and cannot be transferred to the nucleus, resulting in impaired tumor suppressive ability of PTEN in human glioblastoma [16,22].

\section{The effects of UVB on PTEN and mutant subcellular localization}

Available evidence suggested that UV radiation acts as a complete carcinogen, activating oncogenic signaling pathways [23], and inducing tumors by damaging DNA [24]. We explored the effect of UV radiation on the PTEN protein subcellular localization in the well-established WT-PTEN expressing U87MG cells and K13E-PTEN expressing U87MG cells to determine whether UV is a key factor of PTEN cytoplasmic-nuclear translocation. When the cells were continued to be cultured for $24 \mathrm{~h}$ after exposing to the increasing doses of UV radiation $\left(0,90,180,270 \mathrm{~mJ} / \mathrm{cm}^{2}\right)$, nuclear translocation of K13E-PTEN was increased gradually in a UV dose-dependent manner, reaching the highest levels after $270 \mathrm{~mJ} / \mathrm{cm}^{2}$ exposure (Fig. 3A). Similar results were observed for the WT-PTEN in U87MG cells that UV irradiation could also promote nuclear localization of WT-PTEN in a dose-dependent manner (Fig. 3B).

Next, we considered whether it's a universal process in various kinds of cells for UV irradiation resulting in an increase in PTEN nuclear translocation. For this regard,

HEK293T cells transiently-transfected with pEGFP-WT-PTEN or pEGFP-K13E-PTEN vector were exposed to the different doses of UV radiation $\left(0,90,180,270 \mathrm{~mJ} / \mathrm{cm}^{2}\right)$ respectively, and cytoplasm/nuclei fractionation techniques and fluorescence microscopy analysis were performed after $6 \mathrm{~h}$ ( Fig. 4A and $\mathrm{C}$ ) or $12 \mathrm{~h}$ (Fig. 4B and D) post-UVB culture. Similar to the case of U87MG cells, UVB produced a marked increase in both nuclear K13E-PTEN and WT-PTEN levels when the dose was equal or greater than 180 $\mathrm{mJ} / \mathrm{cm}^{2}$, and reaching the highest levels at $270 \mathrm{~mJ} / \mathrm{cm}^{2}$ (Fig. 4E and F). And moreover, WT-PTEN had a higher level of nuclear accumulation after inducing by UV-irradiation, compared to the K13E-PTEN. Taken together, the results confirmed that UVB was indeed a powerful regulator of PTEN nuclear-translocation, even it could reverse K13E mutation induced PTEN cytoplasm accumulation.

\section{Increased PTEN nuclear translocation contributes to UVB injuries}

We investigated whether PTEN nuclear translocation can augment UVB-induced injuries. Different groups of U87MG cells were exposed to UVB with the dose range from 0-960 mJ/cm², and cell viability assay was analyzed after 24h post-UVR culture. As illustrated in Fig. 5A, dose-response curves of parental and vector control U87MG cells showed quite similar curves. While, both PTEN-expressing U87MG cells and K13E-PTEN expressing U87MG cells displayed a markedly enhanced radiation sensitive compared to the 
vector control cells. And in contrast, WT-PTEN expressing U87MG cells had a more sensitive than K13EPTEN expressing U87MG cells. These findings were related with the alterations of the apoptosis signal pathway. After $24 \mathrm{~h}$ post-UVR culture, the UVB resulted in extremely significant increase in the level of cleaved-caspase-3 in each group (Fig. 5B). There was no significant difference between the parental and vector control U87MG cells. However, a higher expression level of cleaved-caspase-3 was observed in WTPTEN expressing U87MG cells and K13E-PTEN expressing U87MG cells, compared to the vector control group. Thus, the above results demonstrated that nuclear translocation of PTEN was critical to its tumorsuppressing functions that made U87MG cells more sensitive to the UV irradiation.

\section{Discussion}

PTEN, a tumor suppressor gene, has lipid phosphatase and protein phosphatase activities, which inhibits the proliferation, invasion and migration of tumor cells. In addition, different distributions of PTEN in the nucleus and cytoplasm reflect the different functions. Nuclear PTEN plays an important role in repairing DNA damage and maintaining chromosome stability or heterochromatin structure [4,7], which enhances the tumor-suppressive effect by adjusting assembly and activity of the APC-CDH1 complex [25]. Therefore, the subcellular localization of PTEN is directly related to the cell final destiny. In this paper, it mainly investigated how would the PTEN complete nuclear and cytoplasm distribution and whether PTEN nucleus translocation affected the biological function of tumor cells under ultraviolet irradiation.

Firstly, two kinds of cells were selected including HeLa and U87MG cells which both lacked expression of PTEN protein. Notably, U87MG cells innately lacked PTEN expression while PTEN-deficient HeLa cells were constructed in our laboratory by CRISPR. The results showed that the two cell models have different sensitivity to UV radiation. PTEN-deficient HeLa cells showed strong radiation resistance, by which the cells had no obvious damage exposed to different doses of UVB (the results are not shown in this paper). While on the contrary, U87MG cells showed a significant radiation sensitivity in a dose-dependent manner. Therefore, U87MG cells were finally selected as a research object to construct WT-PTEN and K13E-PTEN overexpressing cell models for studying whether UV is an effective factor of PTEN cytoplasmic-nuclear translocation, and whether PTEN nuclear translocation can affect UVB-induced injuries.

In our study, the result showed that the WT-PTEN was expressed both in cell nucleus and cytoplasm, and mainly presented in the nucleus, while in the K13E -PTEN expressing cells, PTEN was primarily presented in the cytoplasm. However, after UVB, nuclear accumulations of WT-PTEN and K13E-PTEN protein both were increased gradually in a UV dose-dependent manner. In addition, similar results were observed in the WT-PTEN/K13E-PTEN expressing HEK293T cells that UV irradiation could also promote nuclear entry of WT-PTEN/K13E-PTEN protein in a dose-dependent manner (Fig 4E and F). It was demonstrated that UVB was indeed a powerful inducer of PTEN nuclear-translocation, and moreover, it is a universal process in different kinds of cells (Fig 3 and Fig 4). The molecular mechanism of PTEN nucleus entry may be closely related to PTEN mono-ubiquitination site such as K13 site, controlling the translocation of PTEN from cytoplasm to nucleus [26]. However, After K13 residue was mutated into E (K13E), PTEN could still 
enter the nucleus after exposed to UVB, for which the accurate underlying mechanism warrants further exploration.

Several publications have been reported a proapoptotic function of nuclear PTEN, that increased apoptotic DNA fragmentation is observed in the co-incubation of NGF-treated PC12 cell nuclei [27-29]. We were curious about what changes can be caused by nuclear translocation of PTEN in cell function? Therefore, after WT-PTEN and K13E-PTEN expressing U87MG cells exposure to UV irradiation, cell viability and apoptosis were further studied. The results showed that for WT-PTEN or K13E-PTEN groups, cell viability was inhibited, and the level of apoptosis signal molecule, cleaved-caspase-3 was increased, leading to an increase in cell apoptosis compared with the control cells (Fig. 5). we suspected that apoptotic pathway enhanced nuclear entry of PTEN, and overexpression of catalytically active nuclear PTEN to form the positive feedback against proapoptotic responses in U87MG. Our findings were consistent with the publication that apoptotic pathway enhanced radiation sensitive. Sun and her colleagues showed that overexpression PTEN in the PTEN-deficient cell line PC3 made cells more sensitive to radio-toxicity [30]. Thus, in our study we demonstrated that nuclear translocation of PTEN was critical to its tumor-suppressing functions that made U87MG cells more sensitive to the UV irradiation. Furthermore, with different doses of UVB treatment, PTEN functions may also be different in various cell types via different mechanisms. In skin carcinogenesis, low-dose UVB radiation (no more than $30 \mathrm{~mJ} / \mathrm{cm}^{2}$ ), PTEN positively regulates UVB-induced DNA damage repair in an AKT-independent manner [19]. Likewise, upon $90 \mathrm{~mJ} / \mathrm{cm}^{2}$ UVB treatment, overexpression of PTEN decreases the level of UVB-induced apoptosis in retinal pigment epithelium cells by suppressing $\mathrm{p}-\mathrm{H} 2 \mathrm{~A}$ in response to DNA damage [18], while there might be no direct relationship between the expression of endogenous PTEN and the extent of apoptosis. Thus, there are several potential regulation mechanisms of PTEN signaling following UVB irradiation. We speculated that PTEN plays important role in DNA damage repair following a low dose of UVB radiation, thereby protecting cells against UVB-induced apoptosis. However, a high dose of UVB radiation induces an increased nuclear accumulation of PTEN that may contribute to more complex regulatory functions of PTEN in UV irradiation, such as promoting apoptosis. Taken together, further precise mechanistic studies are needed in order to understand the role of PTEN played in UVB irradiation.

\section{Conclusion}

In conclusion, the results showed that PTEN enhanced the radiation sensitivity of U87MG cells. After irradiation, PTEN entered the cell nucleus, inhibited cell proliferation and promoted cell apoptosis, leading to increased irradiation damage of U87MG cells. It was the first report to demonstrate that UVB can promote PTEN translocation and further affected the function of PTEN, which provided a theoretical basis for studying the role of PTEN in nucleus and cytoplasm. However, the specific mechanism about how the PTEN enters the nucleus under ultraviolet radiation needs further study.

\section{Abbreviations}


UVR, Ultraviolet Radiation; WT-PTEN, Wild-type-PTEN;PI3K, phosphatidylinositol 3' kinase; UVB, ultraviolet radiation b; RPE, retinal pigment epithelium; FBS, Fetal Bovine Serum; DAPI, 4,6-diamino-2-phenyl indole

\section{Declarations}

\section{Ethics Declarations}

Ethical Approval and Consent to participate

Not applicable.

\section{Consent for publication}

The authors agree with publication.

\section{Competing interests}

The authors declare that they have no conflict of interests.

\section{Availability of supporting data}

Not applicable.

\section{Funding}

This work was supported by the National Natural Science Foundation of China (No.31371386) and Natural Science Foundation of Henan Province (No.162300410042).

\section{Contributions}

In this study, WZ and CY performed the experiments, analyzed the data and wrote the manuscript. YA contributed to the experiments. YJ, ZS and LS assisted with data analysis. FW, SJ and LM conceived the idea, supervised the experiments and revised the manuscript.

\section{Acknowledgements}

The authors thank the National Natural Science Foundation of China and Natural Science Foundation of Henan Province of Shaoping Ji this study.

\section{References}

[1] Gimm, O., Perren, A., Weng, L.P. et al. (2000). Differential nuclear and cytoplasmic expression of PTEN in normal thyroid tissue, and benign and malignant epithelial thyroid tumors. Am J Pathol 156, 1693-700. 
[2] Perren, A., Komminoth, P., Saremaslani, P. et al. (2000). Mutation and expression analyses reveal differential subcellular compartmentalization of PTEN in endocrine pancreatic tumors compared to normal islet cells. Am J Pathol 157, 1097-103.

[3] Whiteman, D.C., Zhou, X.P., Cummings, M.C. et al. (2002). Nuclear PTEN expression and clinicopathologic features in a population-based series of primary cutaneous melanoma. Int $J$ Cancer 99 , 63-7.

[4] Ho, J., Cruise, E.S., Dowling, R.J.O. and Stambolic, V. (2020). PTEN Nuclear Functions. Cold Spring Harb Perspect Med 10

[5] Carracedo, A., Alimonti, A. and Pandolfi, P.P. (2011). PTEN level in tumor suppression: how much is too little? Cancer Res 71, 629-33.

[6] Bononi, A. and Pinton, P. (2015). Study of PTEN subcellular localization. Methods 77-78, 92-103.

[7] Planchon, S.M., Waite, K.A. and Eng, C. (2008). The nuclear affairs of PTEN. J Cell Sci 121, 249-53.

[8] Salmena, L., Carracedo, A. and Pandolfi, P.P. (2008). Tenets of PTEN tumor suppression. Cell 133, 403-14.

[9] Trotman, L.C., Wang, X., Alimonti, A. et al. (2007). Ubiquitination regulates PTEN nuclear import and tumor suppression. Cell 128, 141-56.

[10] Malaney, P., Palumbo, E., Semidey-Hurtado, J. et al. (2018). PTEN Physically Interacts with and Regulates E2F1-mediated Transcription in Lung Cancer. Cell Cycle 17, 947-962.

[11] Liu, F., Wagner, S., Campbell, R.B. et al. (2005). PTEN enters the nucleus by diffusion. J Cell Biochem 96, 221-34.

[12] Gil, A., Andrés-Pons, A., Fernández, E. et al. (2006). Nuclear localization of PTEN by a Ran-dependent mechanism enhances apoptosis: Involvement of an N-terminal nuclear localization domain and multiple nuclear exclusion motifs. Mol Biol Cell 17, 4002-13.

[13] Chung, J.H., Ginn-Pease, M.E. and Eng, C. (2005). Phosphatase and tensin homologue deleted on chromosome 10 (PTEN) has nuclear localization signal-like sequences for nuclear import mediated by major vault protein. Cancer Res 65, 4108-16.

[14] Wang, X., Trotman, L.C., Koppie, T. et al. (2007). NEDD4-1 is a proto-oncogenic ubiquitin ligase for PTEN. Cell 128, 129-39.

[15] Bassi, C., Ho, J., Srikumar, T. et al. (2013). Nuclear PTEN controls DNA repair and sensitivity to genotoxic stress. Science 341, 395-9. 
[16] Walker, S.M., Leslie, N.R., Perera, N.M., Batty, I.H. and Downes, C.P. (2004). The tumour-suppressor function of PTEN requires an N-terminal lipid-binding motif. Biochem J 379, 301-7.

[17] Pacholczyk, M., Czernicki, J. and Ferenc, T. (2016). [The effect of solar ultraviolet radiation (UVR) on induction of skin cancers]. Med Pr67, 255-66.

[18] He, J., Long, C., Huang, Z. et al. (2017). PTEN Reduced UVB-Mediated Apoptosis in Retinal Pigment Epithelium Cells. Biomed Res Int 2017, 3681707.

[19] Ming, M., Feng, L., Shea, C.R. et al. (2011). PTEN positively regulates UVB-induced DNA damage repair. Cancer Res 71, 5287-95.

[20] Cheng, X.S., Li, M.S., Du, J. et al. (2011). Neuronal apoptosis in the developing cerebellum. Anat Histol Embryol 40, 21-7.

[21] Huang, Y., Zhou, J., Huang, Y. et al. (2016). SARI, a novel target gene of glucocorticoid receptor, plays an important role in dexamethasone-mediated killing of B lymphoma cells. Cancer Lett 373, 57-66.

[22] Georgescu, M.M., Kirsch, K.H., Kaloudis, P. et al. (2000). Stabilization and productive positioning roles of the C2 domain of PTEN tumor suppressor. Cancer Res 60, 7033-8.

[23] Bowden, G.T. (2004). Prevention of non-melanoma skin cancer by targeting ultraviolet-B-light signalling. Nat Rev Cancer 4, 23-35.

[24] Setlow, R.B. (1974). The wavelengths in sunlight effective in producing skin cancer: a theoretical analysis. Proc Natl Acad Sci U S A 71, 3363-6.

[25] Song, M.S., Carracedo, A., Salmena, L. et al. (2011). Nuclear PTEN regulates the APC-CDH1 tumorsuppressive complex in a phosphatase-independent manner. Cell 144, 187-99.

[26] Zhang, S., Taghibiglou, C., Girling, K. et al. (2013). Critical role of increased PTEN nuclear translocation in excitotoxic and ischemic neuronal injuries. J Neurosci 33, 7997-8008.

[27] Ahn, J.Y., Rong, R., Liu, X. and Ye, K. (2004). PIKE/nuclear PI 3-kinase signaling mediates the antiapoptotic actions of NGF in the nucleus. Embo j 23, 3995-4006.

[28] Ye, K. (2005). PIKE/nuclear PI 3-kinase signaling in preventing programmed cell death. J Cell Biochem 96, 463-72.

[29] Ye, K. (2006). PIKE GTPase-mediated nuclear signalings promote cell survival. Biochim Biophys Acta $1761,570-6$.

[30] Sun, Y., St Clair, D.K., Fang, F. et al. (2007). The radiosensitization effect of parthenolide in prostate cancer cells is mediated by nuclear factor-kappaB inhibition and enhanced by the presence of PTEN. Mol Cancer Ther 6, 2477-86. 


\section{Figures}

A
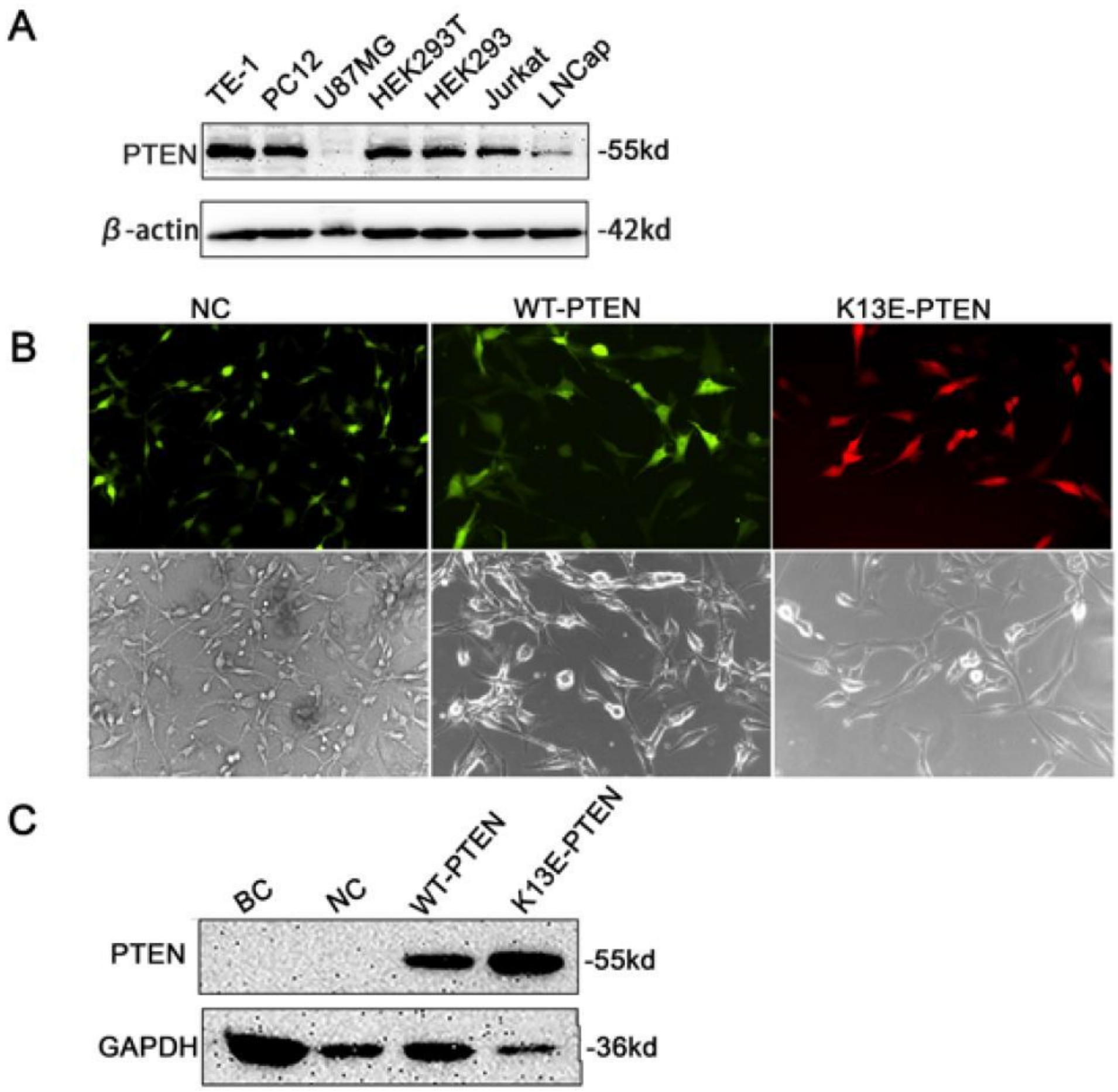

Figure 1

Establishment of WT-PTEN/K13E-PTEN expression in PTEN-deficient U87MG cell lines. (A) PTEN protein in many different cell lines detected by Western blotting. (B) Lentivirus expressing of pGFP-WT-PTEN and mutant pRFP-K13E-PTEN in U87MG cells detected by fluorescence microscopy, $200 \mathrm{x}$, and negative group (NC), 100x. (C) The recombinant wild-type and K13E mutant PTEN protein in U87MG cells detected by 
Western blotting. Wild type U87MG cells and U87MG cells transfected with control lentivirus were termed as blank control (BC) and Negative Control (NC), respectively.

A
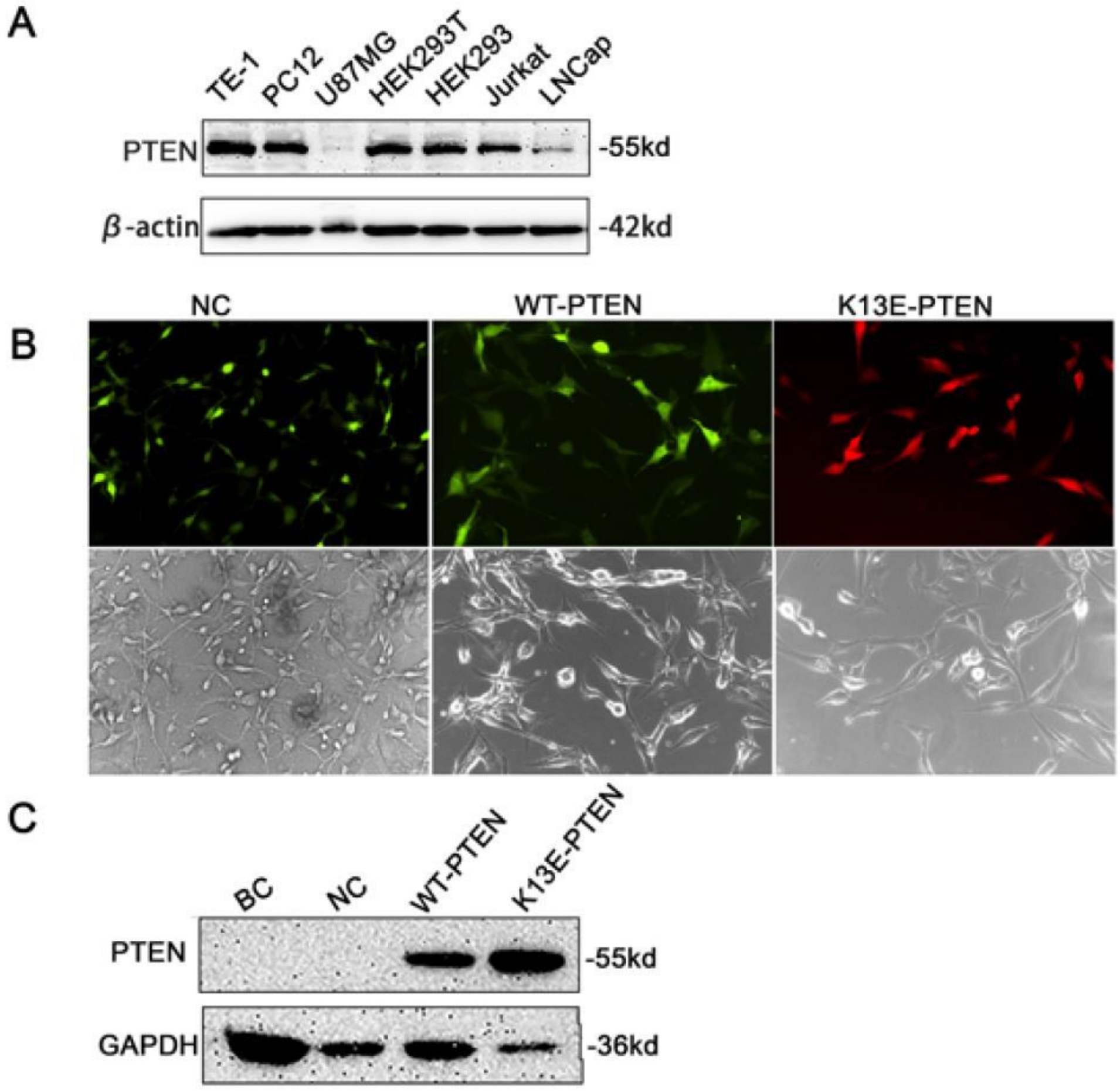

Figure 1

Establishment of WT-PTEN/K13E-PTEN expression in PTEN-deficient U87MG cell lines. (A) PTEN protein in many different cell lines detected by Western blotting. (B) Lentivirus expressing of pGFP-WT-PTEN and mutant pRFP-K13E-PTEN in U87MG cells detected by fluorescence microscopy, $200 \mathrm{x}$, and negative group (NC), 100x. (C) The recombinant wild-type and K13E mutant PTEN protein in U87MG cells detected by 
Western blotting. Wild type U87MG cells and U87MG cells transfected with control lentivirus were termed as blank control (BC) and Negative Control (NC), respectively.

A
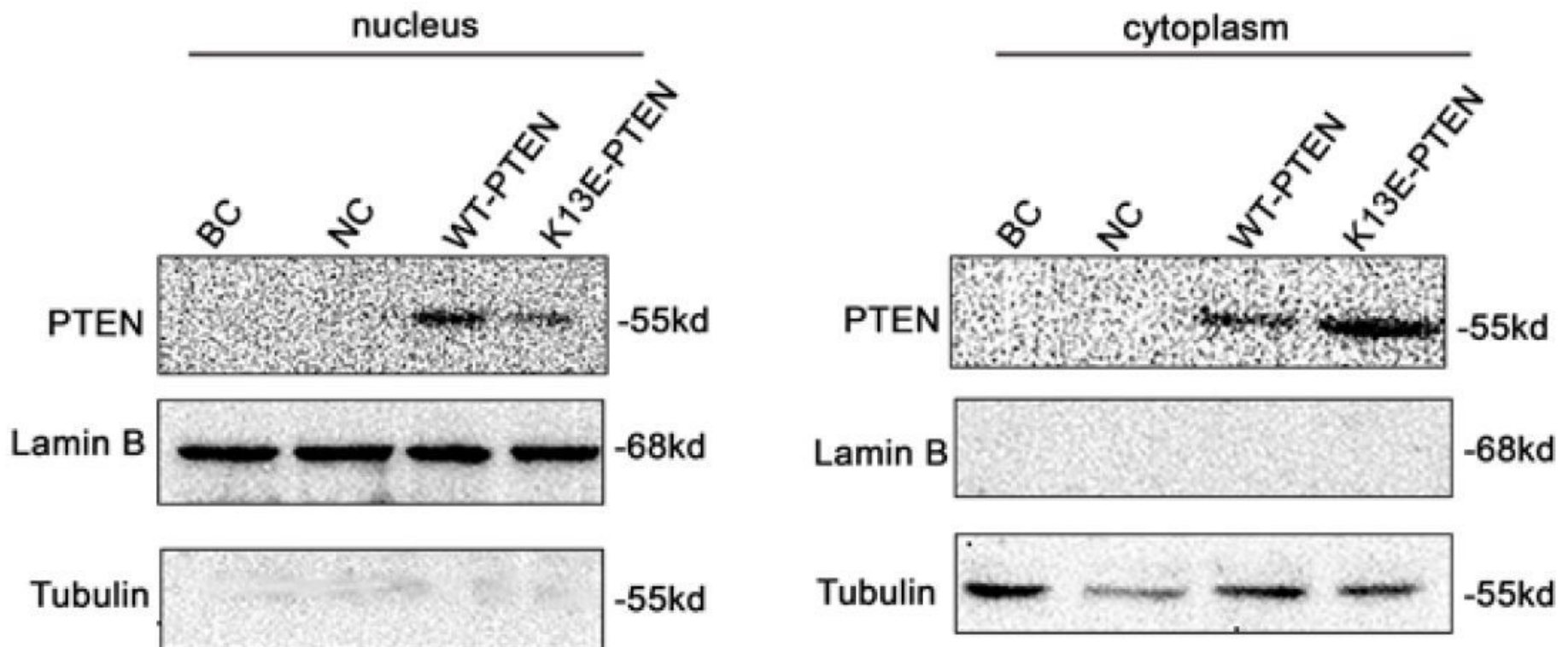

B
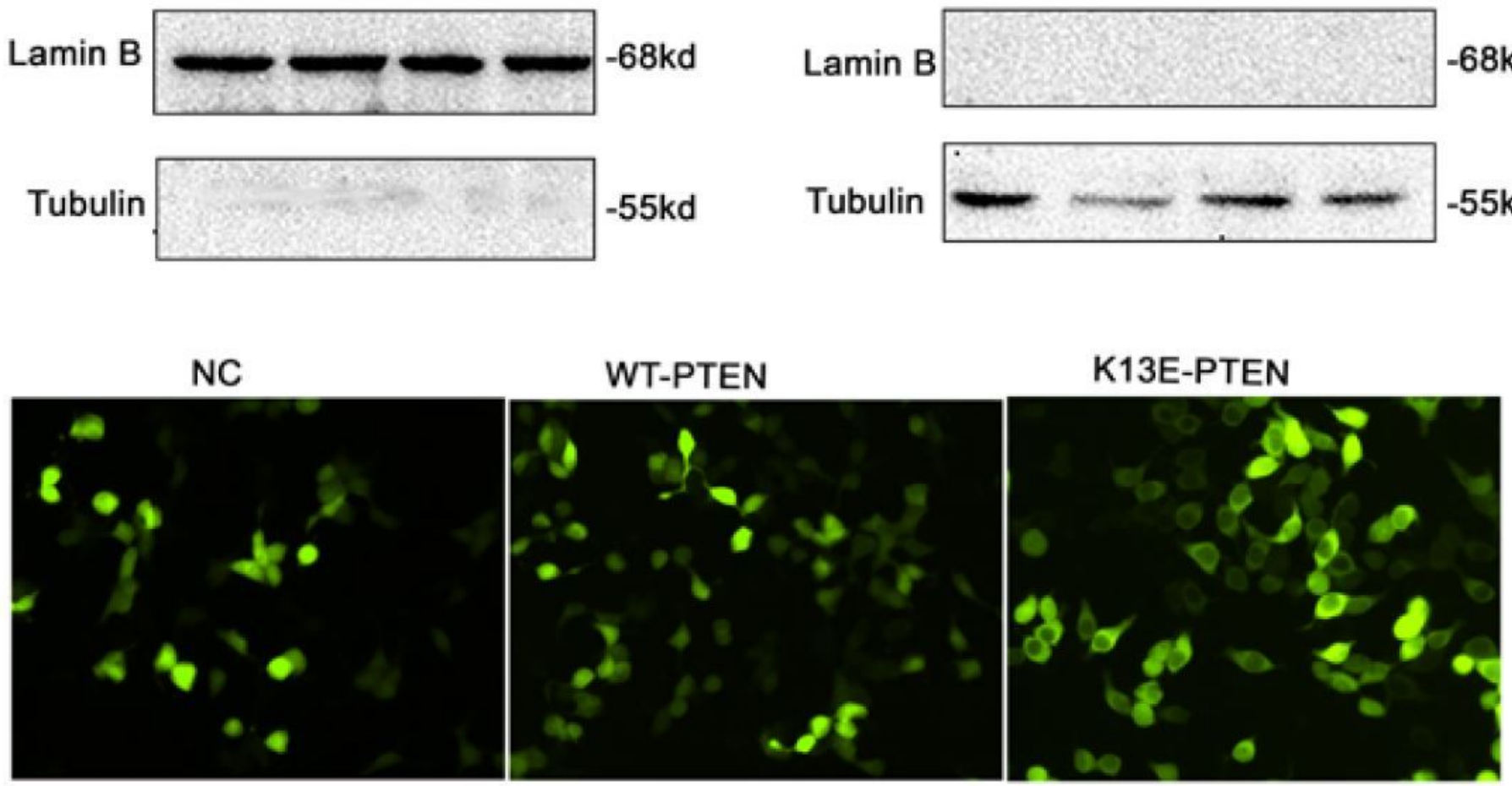

Figure 2

Subcellular localization of PTEN and mutant. (A) The nuclear and cytoplasmic proteins were detected with western blotting in PTEN/ K13E-PTEN overexpressing U87MG cells. WT-PTEN exhibited a distribution in both nuclear and cytoplasmic compartments, the K13E-PTEN mutant was predominantly in cytoplasm.

(B) Fluorescence image of WT-PTEN and K13E-PTEN subcellular localization in HEK293T cells transiently-transfected with pEGFP-WT-PTEN or pEGFP-K13E-PTEN vector, $200 \times$. 

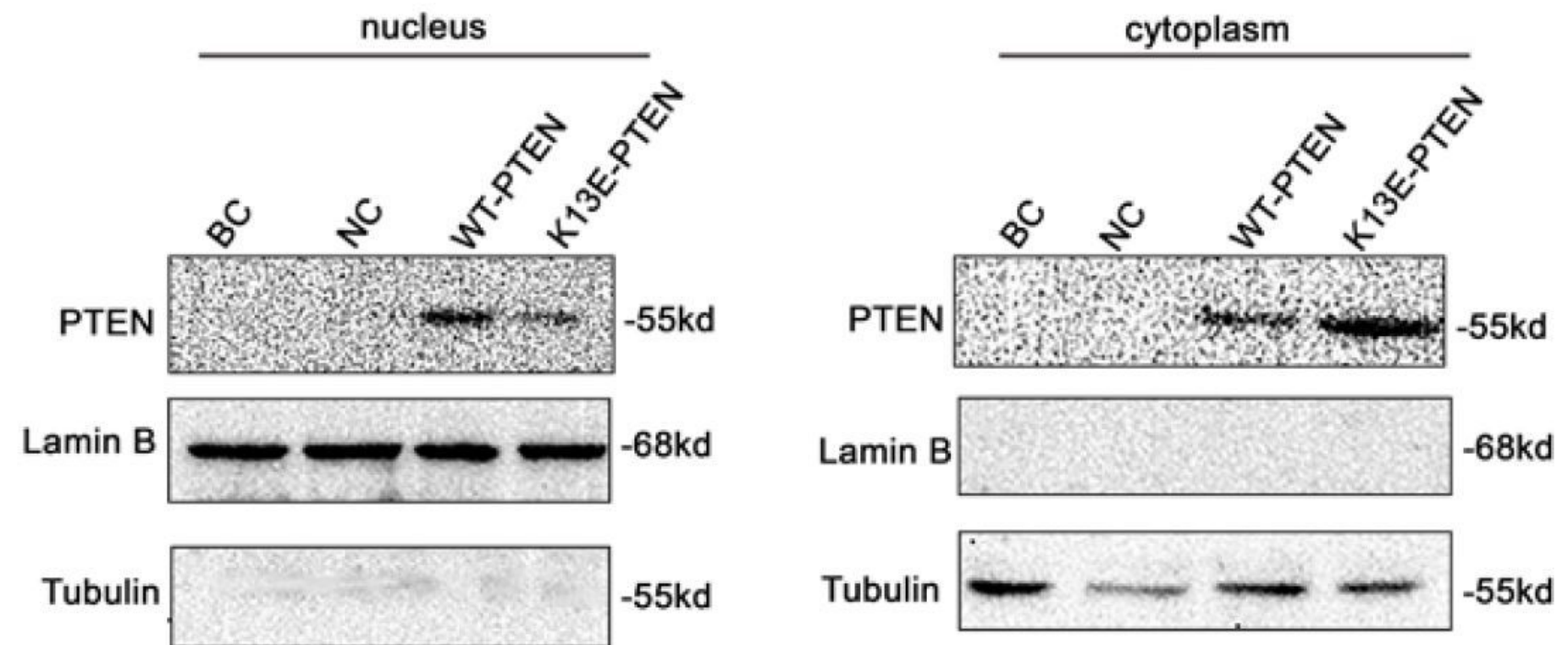

B
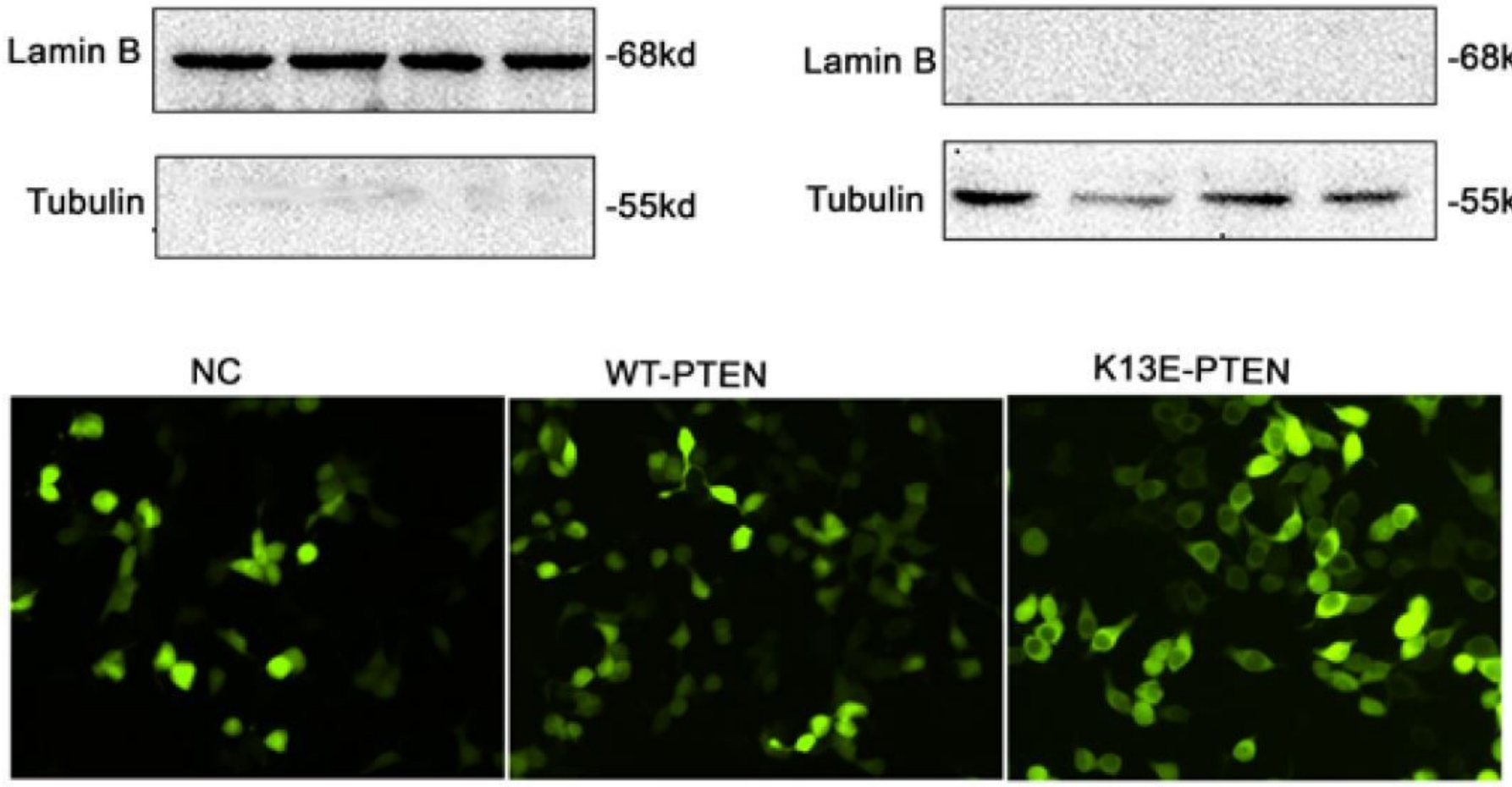

Figure 2

Subcellular localization of PTEN and mutant. (A) The nuclear and cytoplasmic proteins were detected with western blotting in PTEN/ K13E-PTEN overexpressing U87MG cells. WT-PTEN exhibited a distribution in both nuclear and cytoplasmic compartments, the K13E-PTEN mutant was predominantly in cytoplasm. (B) Fluorescence image of WT-PTEN and K13E-PTEN subcellular localization in HEK293T cells transiently-transfected with pEGFP-WT-PTEN or pEGFP-K13E-PTEN vector, $200 \times$.

A

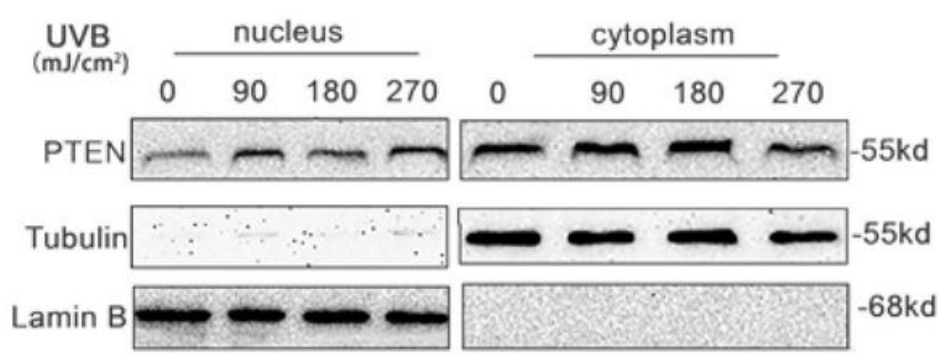

K13E-PTEN
B

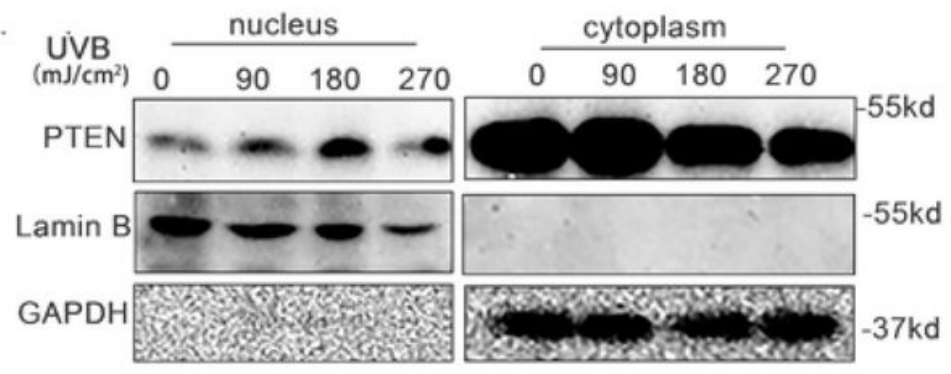

WT-PTEN 


\section{Figure 3}

UVB stress increases nuclear translocation of both PTEN and mutant in PTEN/K13E-PTEN overexpressing U87MG cells. After exposure to the different doses of UV radiation $(0,90,180$, $270 \mathrm{~mJ} / \mathrm{cm} 2)$, U87MG cells continued to be cultured for $24 \mathrm{~h}$, the nuclear translocation of K13E-PTEN (A) and WT-PTEN (B) were detected with western blotting.

A

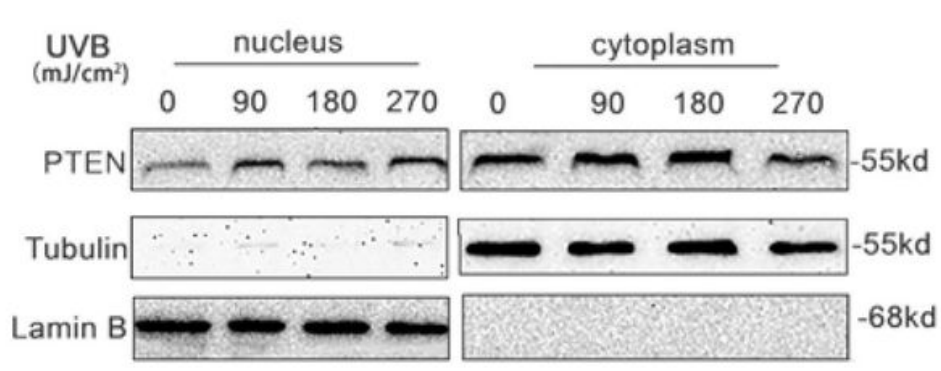

K13E-PTEN
B

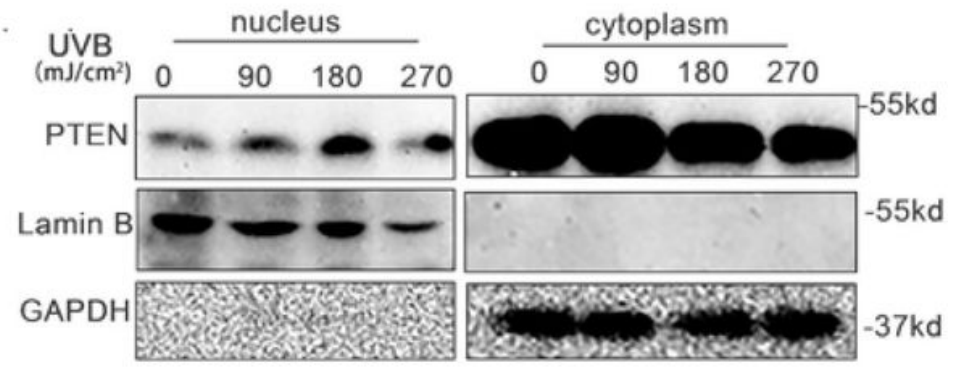

WT-PTEN

\section{Figure 3}

UVB stress increases nuclear translocation of both PTEN and mutant in PTEN/K13E-PTEN overexpressing U87MG cells. After exposure to the different doses of UV radiation $(0,90,180$, $270 \mathrm{~mJ} / \mathrm{cm} 2)$, U87MG cells continued to be cultured for $24 \mathrm{~h}$, the nuclear translocation of K13E-PTEN (A) and WT-PTEN (B) were detected with western blotting. 
A

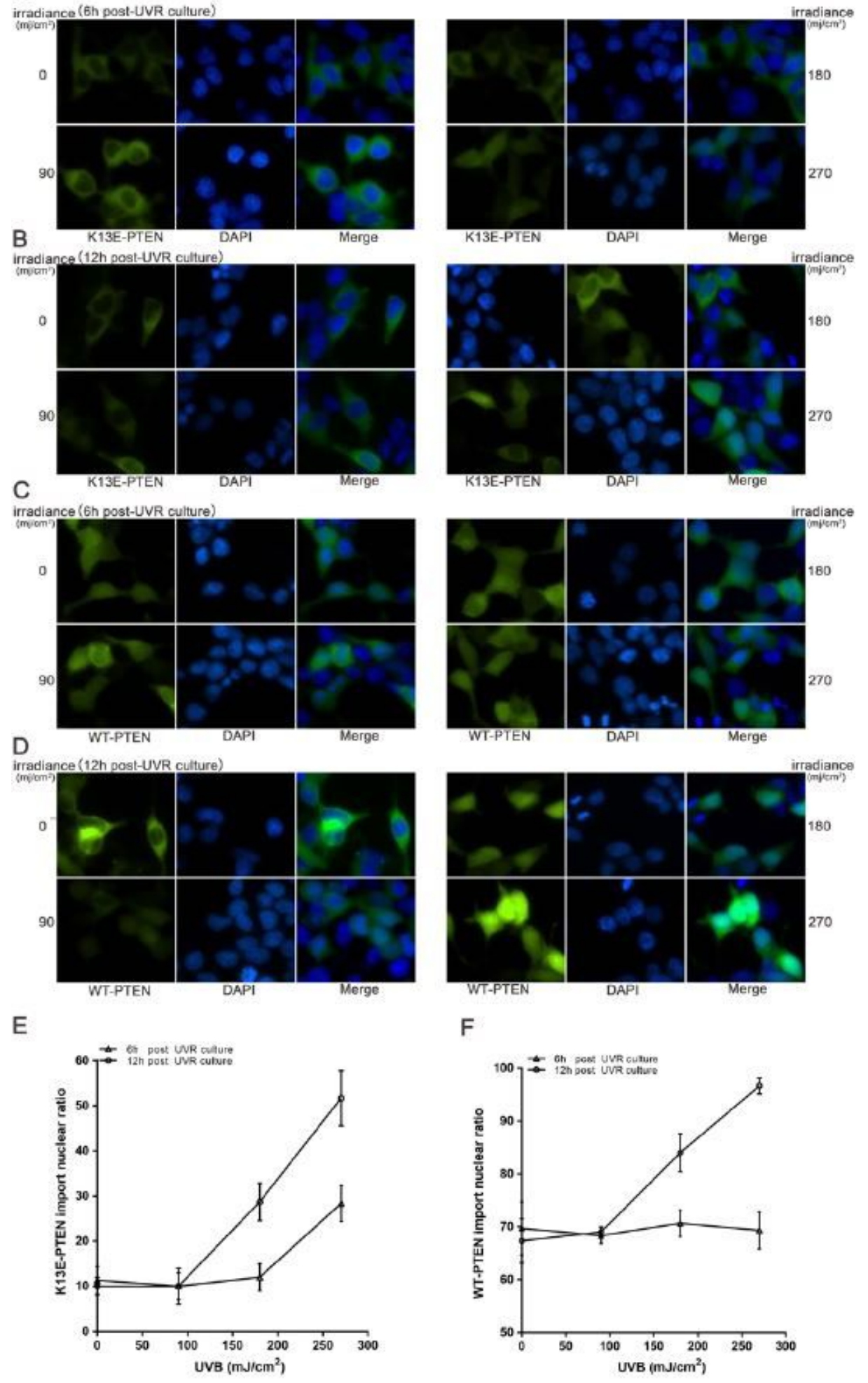

Figure 4

The effects of UVB on nuclear localization of PTEN and mutant in HEK293T cells transiently-transfected with pEGFP-K13E-PTEN and pEGFP-WT-PTEN. After $6 \mathrm{~h}$ (A and C) or $12 \mathrm{~h}$ (B and D) post-UVR culture, K13E-PTEN (A and B) or WT-PTEN (C and D) was photographed by fluorescence microscopy, $200 \times$. The images were shown with DAPI labeling of the nucleus (blue) and PTEN/PTEN-K13E location with GFP (green). And the overlapped blue and green in the merged images represented PTEN localization in the 
nuclear. (E) and (F) The statistics analysis of PTEN nucleus import ratio after exposure to different doses of UVB in WT-PTEN/ K13E-PTEN overexpressing HEK293T cells after $6 \mathrm{~h}$ and $12 \mathrm{~h}$ post-UVR culture, respectively. the percentage of WT-PTEN/K13E-PTEN in the nucleus were counted in at least 50 cells.

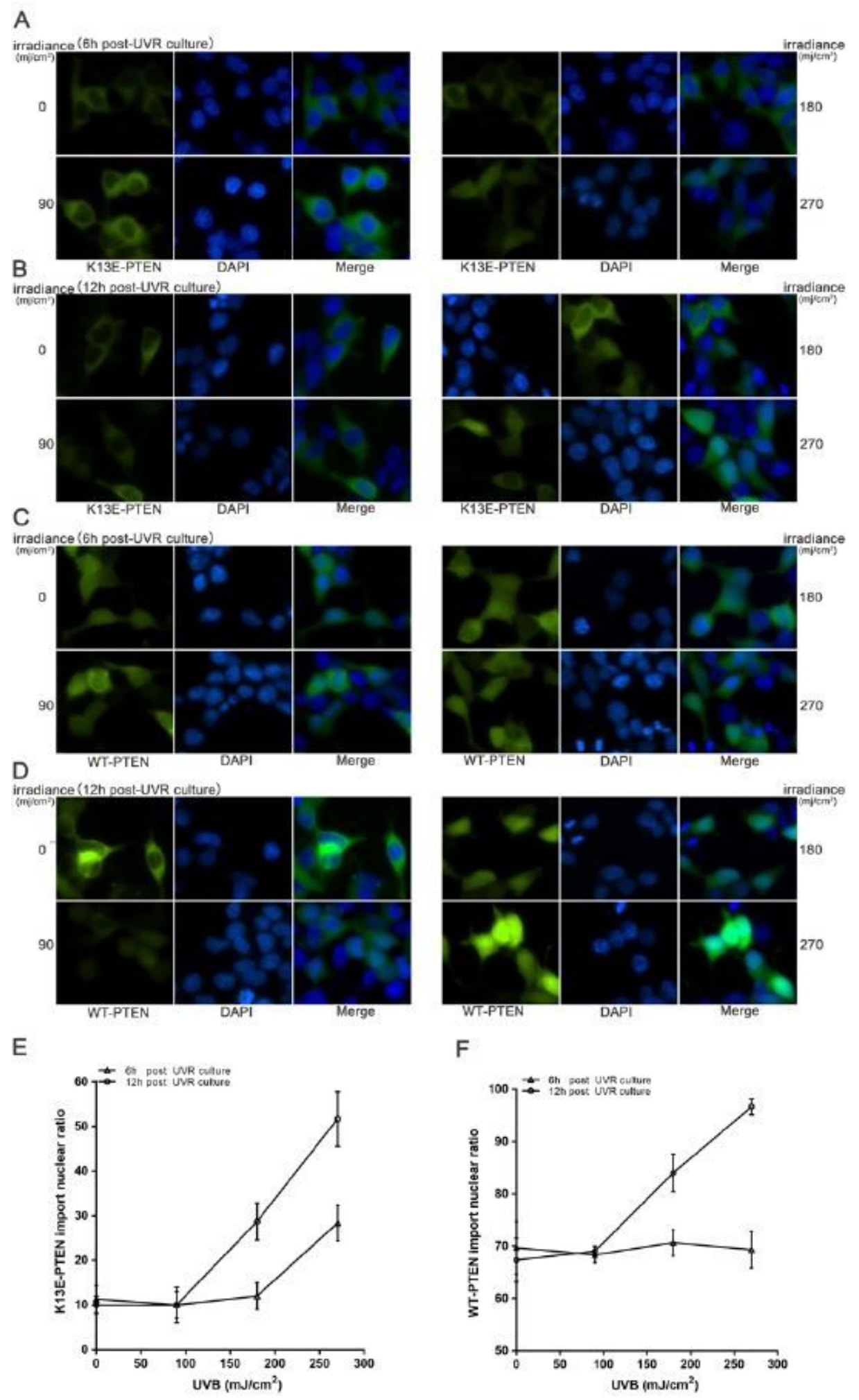

Figure 4

The effects of UVB on nuclear localization of PTEN and mutant in HEK293T cells transiently-transfected with pEGFP-K13E-PTEN and pEGFP-WT-PTEN. After $6 \mathrm{~h}$ (A and C) or $12 \mathrm{~h}$ (B and D) post-UVR culture, 
K13E-PTEN (A and B) or WT-PTEN (C and D) was photographed by fluorescence microscopy, $200 \times$. The images were shown with DAPI labeling of the nucleus (blue) and PTEN/PTEN-K13E location with GFP (green). And the overlapped blue and green in the merged images represented PTEN localization in the nuclear. $(E)$ and $(F)$ The statistics analysis of PTEN nucleus import ratio after exposure to different doses of UVB in WT-PTEN/ K13E-PTEN overexpressing HEK293T cells after $6 \mathrm{~h}$ and $12 \mathrm{~h}$ post-UVR culture, respectively. the percentage of WT-PTEN/K13E-PTEN in the nucleus were counted in at least 50 cells.

A

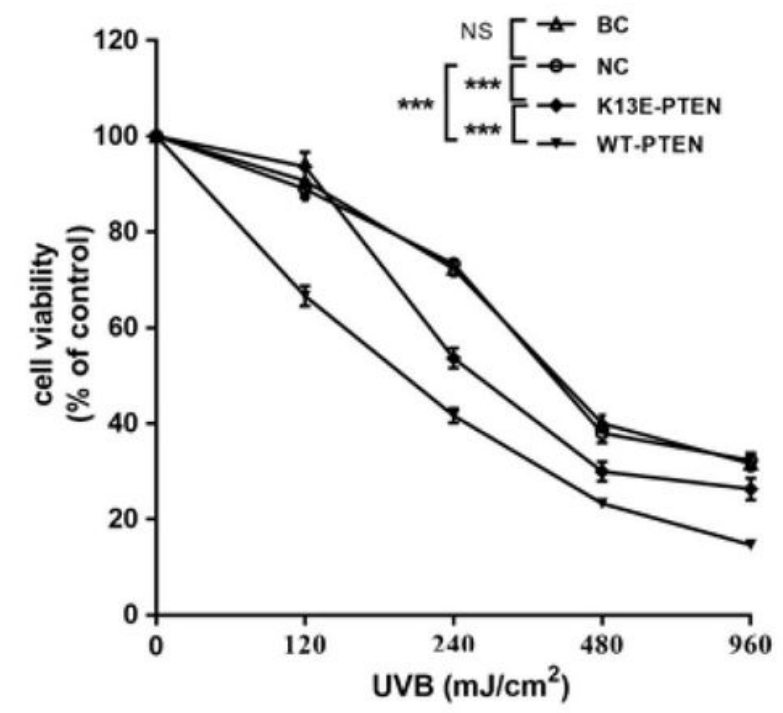

B

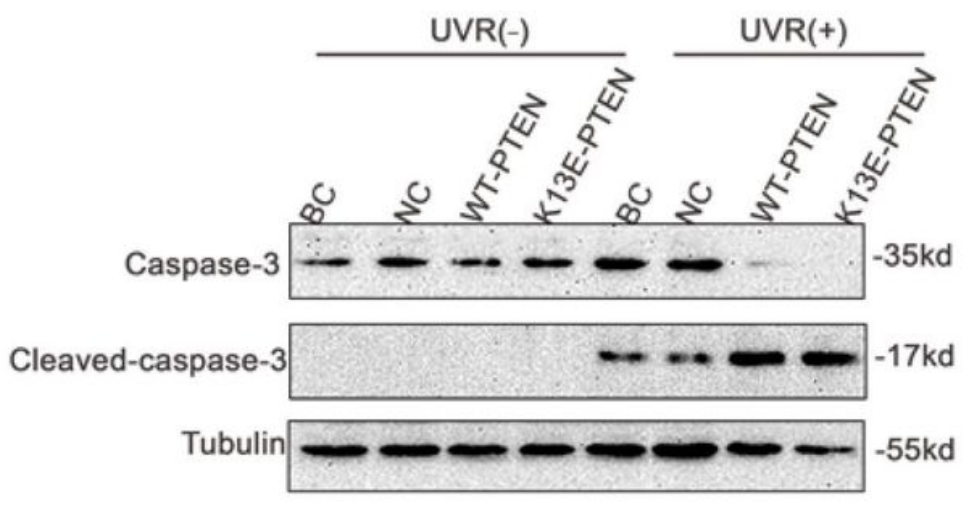

\section{Figure 5}

Overexpression of WT-PTEN/K13E-PTEN enhances UVB-induced damage in U87MG cells. (A) Cell viability was detected by MTT assay after $24 \mathrm{~h}$ post-UVR culture. Values are expressed as mean $\pm S D(* \star * P<0.001$, $t$ test). (B) Western blot analysis shows increased levels of Cleaved-caspase-3 in WT/K13E-PTEN overexpressing U87MG cells compared to the $\mathrm{NC}$ and $\mathrm{BC}$ group after $24 \mathrm{~h}$ post-UVR culture.

A
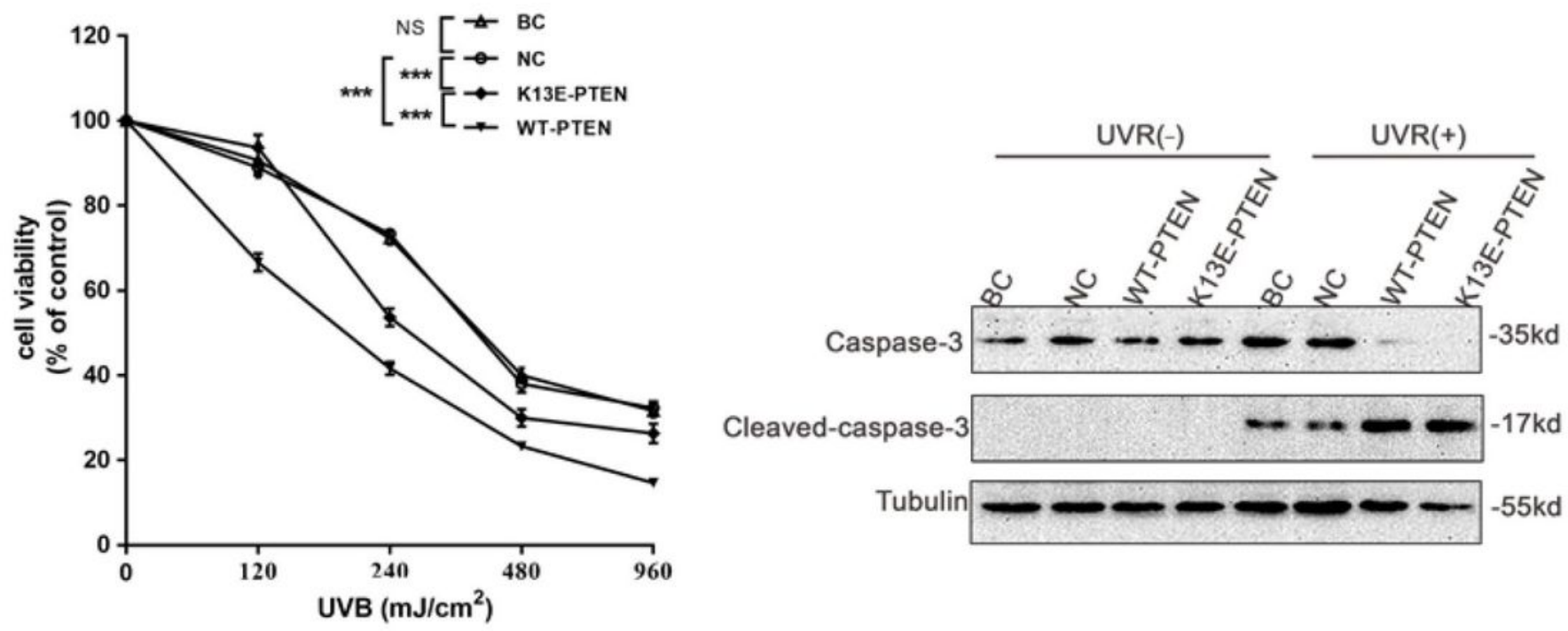

Figure 5 
Overexpression of WT-PTEN/K13E-PTEN enhances UVB-induced damage in U87MG cells. (A) Cell viability was detected by MTT assay after $24 \mathrm{~h}$ post-UVR culture. Values are expressed as mean $\pm S D(\star \star \star P<0.001$, $t$ test). (B) Western blot analysis shows increased levels of Cleaved-caspase-3 in WT/K13E-PTEN overexpressing U87MG cells compared to the NC and BC group after $24 \mathrm{~h}$ post-UVR culture. 